Relations industrielles

Industrial Relations

\title{
Cases in Business Policy, Donald Grunewald, Holt, Rinehart and Winston, New York, 1964, 176 pages.
}

\section{Laurent Bélanger}

Volume 19, numéro 4, 1964

URI : https://id.erudit.org/iderudit/027531ar

DOI : https://doi.org/10.7202/027531ar

Aller au sommaire du numéro

Éditeur(s)

Département des relations industrielles de l'Université Laval

ISSN

0034-379X (imprimé)

1703-8138 (numérique)

Découvrir la revue

Citer ce compte rendu

Bélanger, L. (1964). Compte rendu de [Cases in Business Policy, Donald

Grunewald, Holt, Rinehart and Winston, New York, 1964, 176 pages.] Relations

industrielles / Industrial Relations, 19(4), 528-528.

https://doi.org/10.7202/027531ar

Tous droits réservés (C Département des relations industrielles de l'Université Laval, 1964
Ce document est protégé par la loi sur le droit d'auteur. L'utilisation des services d'Érudit (y compris la reproduction) est assujettie à sa politique d'utilisation que vous pouvez consulter en ligne.

https://apropos.erudit.org/fr/usagers/politique-dutilisation/ 
Cases in Business Policy, Donald Grunewald, Holt, Rinehart and Winston, New York, 1964,176 pages.

Voici un recueil de «cas» touchant des problèmes administratifs généroux au sein des petites, moyennes et grandes entreprises.

Un grand nombre de ces cas portent sur des sujets précis comme l'établissement ou l'amélioration des structures organisationnelles, la formation des cadres, les relations entre les objectifs d'une entreprise et les buts personnels des dirigeants, entre l'entreprise et le milieu social dans lequel elle opère. Ce volume a le mérite de présenter des problèmes qui se situent tant au niveau de la moyenne que de la haute direction. C'est ce qui le différencie des autres «cose books » en administration.

\section{Laurent Bélanger}

Le métier de directeur, Louis $A$. Allen, Les Editions d'Organisation, Paris, 1964, 189 pages.

Ceci est une traduction du volume oméricain: \&anagement as a Profession \$. On y retrouve une description simple et concise de lo tôche d'un directeur d'entreprise. Cette tôche comprend les dimensions suivantes: lo prise de décision, l'établissement des communications, la motivation des subordonnés, le choix et la formotion d'une équipe. De plus, le directeur doit aussi planifier, organiser et contrôler. Lo description des composantes de la tâche s'accompagne des principes administratifs dérivés de l'expérience et formulés d'une façon laconique. Un nombre imposant de conseils touchant l'établissement et la maintenance de rapports mutuels efficaces vient compléter cette description. Sur ce point, l'auteur aurait pu intituler cette section en utilisant le vocable célèbre: * Faites-ça et vous vivrez \$.

La plupart des volumes sur les fondements en administration présente la tâche de l'odministrateur selon la séquence linéai. re suivante: planification, organisation, direction et contrôle. Au lieu de s'attacher ou principe d'analyse logique, l'auteur a opté pour une présentation selon le principe de l'action, en l'occurence, l'accomplissement simultané des quatre grandes composantes de la tâche. Cette opproche lui permet de mettre en relief l'acte même de diriger et de démontrer toute son importance.
La lecture de ce volume fournirait un bon point de départ à un étudiant ou un directeur d'entreprise qui aurait I'intention de parfaire ses connaissances en administration.

\section{Laurent Bélanger}

\section{Applied Business and Economic Statistics,}

par Ya-Lun Chou, Holt, Rinehart and Winston, New York, 1963, 634 poges.

Ce livre s'adresse ovant tout aux étudiants d'un cours plutôt formel de stotistique. Une partie couvre la statistique descriptive traditionnelle. Elle comprend prin. cipalement la collection et la présentation des données, les distributions de fréquence, les mesures de tendance centrale, de voriation et de dispersion, la probabilité et les distributions de probabilité, les distributions binomiale et normale. Un lecteur attentif peut comprendre assez facilement par luimême le contenu de ces différents chapitres.

Mais en plus de la statistique descriptive, le volume du professeur Chou, inclut un bon exposé de l'induction statistique. L'induction, qui constitue l'essentiel de lo statistique, présente de plus grandes difficultés de compréhension que la partie précédente, du moins pour le lecteur non suffisamment overti.

$\mathrm{Ce}$ livre traite égolement d'autres sujets d'intérêt tels que la régression et la corrélation, les nombres-indices, l'analyse des séries temporelles, les mesures de croissance à long terme et de fluctuations à court terme, ainsi que des techniques de projection.

L'auteur s'exprime clairement et la séquence des sujets est logique. De plus, le livre couvre à peu près tous les aspects de la statistique; il se situe, de façon générale, ou niveau de l'introduction à la statistique.

Enfin, le titre de ce livre, «Applied Business and Economic Statistics $\gg$, peut porter à croire qu'il est bâti en fonction d'abord des décisions des administrateurs de l'entreprise. En fait, d'après son contenu, il s'apparente très fortement oux volumes conventionnels en statistique. Ce qui le rend plus utile aux administrateurs, c'est à peu près uniquement le fait que de nombreux exemples du monde des affaires illustrent I'applicabilité de la statistique à l'éclairage des décisions administratives. Mais il existe par ailleurs d'outres volumes qui présentent systémotiquement des méthodes quantitati- 